\title{
Estimation of exponential sums of polynomials of higher degrees II
}

\author{
by
}

YAngbo Ye (Iowa City, IA)

1. Introduction. In Ye [10] the author proved the following bounds for an exponential sum. Let $p$ be an odd prime and let $b$ and $c$ be integers relatively prime to $p$. Set $q=p^{a}, a \geq 1$, and $k \geq 0$. Define the exponential sum

$$
S_{k}(q, b, c)=\sum_{x \bmod q} \mathrm{e}\left(\frac{b x+c x^{k}}{q}\right)
$$

where $\mathrm{e}(x)=e^{2 \pi i x}$. Then for $1<m<p$ we have [10]

$$
\left|S_{\phi(q)-m}(q, b, c)\right| \leq \begin{cases}(m+1) p^{1 / 2}+1 & \text { if } m>1, m \mid(p-1), \text { and } a=1, \\ (m+1) q^{1 / 2} & \text { if } 1<m<p-1 \text { and } a \geq 2, \\ p^{1 / 2} q^{1 / 2} & \text { if } m=p-1 \text { and } a \geq 5, \\ p q^{1 / 2} & \text { if } m=p-1 \text { and } a=4, \\ p^{1 / 2} q^{1 / 2} & \text { if } m=p-1 \text { and } a=3, \\ q^{1 / 2} & \text { if } m=p-1 \text { and } a=2 .\end{cases}
$$

In this article we will prove certain identities between the above exponential sum and hyper-Kloosterman sums, generalize the above estimation for the exponential sum to other cases of $m$ when $a \geq 2$, and establish new bounds for hyper-Kloosterman sums. Write $p^{h} \| n$ if $p^{h} \mid n$ but $p^{h+1} \nmid n$.

Theorem 1. Let $p$ be a prime, $q=p^{a}, a \geq 2$, and $k$ an integer with $a \leq k<\phi(q)$ and $p \nmid k$. We set $h$ by $p^{h} \|(k-1)$. Then for any $b$ and $c$ relatively prime to $p$ we have

$$
\left|S_{k}(q, b, c)\right| \leq \begin{cases}(\phi(q)-k+1) q^{1 / 2} & \text { if } p \nmid(k-1), \\ (\phi(q)-k+1) p^{-h / 2} q^{1 / 2} & \text { if } h \geq 1 \text { and } a \geq 3 h+2, \\ (k-1, p-1) p^{\min (h, a / 2-1)} q^{1 / 2} & \text { if } h \geq 1 \text { and } 2 \mid a, \\ (k-1, p-1) p^{\min (h+1 / 2, a / 2-1)} q^{1 / 2} & \text { if } h \geq 1 \text { and } 2 \nmid a,\end{cases}
$$

2000 Mathematics Subject Classification: Primary 11L07, 11L05.

Supported in part by NSF Grant \#DMS 97-01225. 
when $p>2$, and

$$
\left|S_{k}(q, b, c)\right| \leq \begin{cases}(\phi(q)-k+1) p^{1-h / 2} q^{1 / 2} & \text { if } h \geq 1 \text { and } a \geq 3 h+5, \\ p^{\min (h+1, a / 2-1)} q^{1 / 2} & \text { if } h \geq 1 \text { and } 2 \mid a \\ p^{\min (h+3 / 2, a / 2-1)} q^{1 / 2} & \text { if } h \geq 1 \text { and } 2 \nmid a\end{cases}
$$

when $p=2$.

When $a \geq 3 h+2$ with $p>2$ and when $a \geq 3 h+5$ with $p=2$, two bounds are given in Theorem 1; the smaller bound applies. Loxton and Smith [5] proved that

$$
\left|S_{k}(q, b, c)\right| \leq q^{1 / 2} d_{k-1}(q)(\Delta, q)^{1 / 2}
$$

when $b$ and $c$ are relatively prime to $p$, where $d_{k-1}(q)$ is the number of representations of $q$ as a product of $k-1$ positive integers and $\Delta$ is the discriminant of the derivative of the polynomial $b x+c x^{k}$. After an improvement by Loxton and Vaughan [6], Dąbrowski and Fisher established in [1] better bounds for exponential sums of this kind. Under the restriction of $p \nmid k$, which is the case we will deal with in this paper, their Theorem 1.8 implies the following estimates (see Section 4 for details).

Theorem 2. Let $p$ be a prime, $a \geq 2, q=p^{a}, k \geq 2, p \nmid k$, and $p^{h} \|(k-1)$. Then for any integers $b$ and $c$ relatively prime to $p$ we have

$\left|S_{k}(q, b, c)\right|$

$$
\leq \begin{cases}(k-1) q^{1 / 2} & \text { if } p \nmid(k-1) \text { and } a \geq 2, \\ (k-1) p^{-h / 2} q^{1 / 2} & \text { if } h \geq 1 \text { and } a \geq 3 h+2, \\ (k-1, p-1) p^{\min (h, a / 2-1)} q^{1 / 2} & \text { if } h \geq 1 \text { and } 2 \mid a, \\ (k-1, p-1) p^{\min (h+1 / 2, a / 2-1)} q^{1 / 2} & \text { if } h \geq 1 \text { and } 2 \nmid a,\end{cases}
$$

when $p>2$, and

$$
\left|S_{k}(q, b, c)\right| \leq \begin{cases}(k-1) p^{1-h / 2} q^{1 / 2} & \text { if } h \geq 1 \text { and } a \geq 3 h+5, \\ p^{\min (h+1, a / 2-1)} q^{1 / 2} & \text { if } h \geq 1 \text { and } 2 \mid a \\ p^{\min (h+1 / 2, a / 2-1)} q^{1 / 2} & \text { if } h \geq 1 \text { and } 2 \nmid a\end{cases}
$$

when $p=2$.

We note that the last two cases here for $p>2$ and for $p=2$ are the same as in Theorem 1 . In other cases Theorem 1 is effective for large $k$ while Theorem 2 gives better bounds for small $k$. In particular when $p>2$ and $p \nmid k(k-1)$, we can combine these two theorems and get

$$
\left|S_{k}(q, b, c)\right| \leq \min (k-1, \phi(q)-k+1) q^{1 / 2} .
$$

This estimate becomes worse than trivial when $q^{1 / 2} \leq k \leq \phi(q)-q^{1 / 2}$. What kind of non-trivial bounds one can get for $k$ in this middle range is indeed an interesting question. See Vaughan [8] for a history of estimation of this exponential sum. The question of estimating this exponential sum for large $k$ was posed by Loxton and Vaughan [6]. 
As in [10] our proof of Theorem 1 is based on certain identities between the above exponential sum and hyper-Kloosterman sums (Theorem 3). These identities are in turn deduced from generalized Davenport-Hasse identities of Gauss sums (Theorem 5). Using the new bounds for hyperKloosterman sums for prime power moduli obtained by Dąbrowski and Fisher [1] (see (19), (20), and an improved version in (1) and (2)), we then prove Theorem 1.

We denote a hyper-Kloosterman sum by

$$
K(q, m+1, z)=\sum_{\substack{x_{1}, \ldots, x_{m} \bmod q \\\left(x_{1}, p\right)=\ldots=\left(x_{m}, p\right)=1}} \mathrm{e}\left(\frac{x_{1}+\ldots+x_{m}+z \bar{x}_{1} \ldots \bar{x}_{m}}{q}\right)
$$

for $q=p^{a}, m \geq 1$, and $p \nmid z$. Define an exponential sum by

$$
I(q, m, z)=\sum_{\substack{x \bmod q \\(x, p)=1}} \mathrm{e}\left(\frac{m x+z \bar{x}^{m}}{q}\right) .
$$

The identities for hyper-Kloosterman sums are given in the following theorem. Set $\varepsilon_{p}=1$ if $p \equiv 1(\bmod 4)$, and $\varepsilon_{p}=i$ if $p \equiv 3(\bmod 4)$.

Theorem 3. Let $p$ be a prime, $m \geq 1, p \nmid m, a \geq 2$, and $q=p^{a}$. Then for any integer $z$ with $p \nmid z$ we have

$$
K(q, m+1, z)= \begin{cases}q^{(m-1) / 2} I(q, m, z) & \text { if } 2 \mid a, \\ q^{(m-1) / 2} \varepsilon_{p}^{m-1}\left(\frac{2^{m-1} z^{m-1} m}{p}\right) I(q, m, z) & \text { if } 2 \nmid a,\end{cases}
$$

when $p>2$, and

$$
K(q, m+1, z)=q^{(m-1) / 2}\left(\frac{2}{m}\right)^{a} I(q, m, z)
$$

when $p=2$.

For the case of even $a$ these identities were proved by Smith [7]. When $a=1$ a similar identity is indeed the Diophantine manifestation of a geometric isomorphism of sheaves in Katz [4], Theorem 9.2.3. In Section 3 we will thus only consider the case of odd $a \geq 3$.

To see another application of our identities, we note that for any positive integer $n$,

$$
\begin{aligned}
I(q, m+n \phi(q), z) & =\sum_{\substack{x \bmod q \\
(x, p)=1}} \mathrm{e}\left(\frac{(m+n \phi(q)) x+z \bar{x}^{m+n \phi(q)}}{q}\right) \\
& =\sum_{\substack{y \bmod q \\
(y, p)=1}} \mathrm{e}\left(\frac{m y+z(m+n \phi(q))^{m} \bar{m}^{m} \bar{y}^{m}}{q}\right)
\end{aligned}
$$


where we set $y \equiv(m+n \phi(q)) \bar{m} x(\bmod q)$, which is still relatively prime to $p$ because $p \nmid m, a \geq 2$, and $p \mid \phi(q)$. Since $(m+n \phi(q))^{m} \bar{m}^{m} \equiv 1-n p^{a-1}$ $(\bmod q)$, we have

$$
I(q, m+n \phi(q), z)=I\left(q, m, z\left(1-n p^{a-1}\right)\right) .
$$

Applying this identity to the exponential sums on the right side in Theorem 3, we can easily deduce the following identity for hyper-Kloosterman sums.

COROLlary. Let $p$ be any prime, $m$ and $n$ any positive integer, $p \nmid m$, $a \geq 2$, and $q=p^{a}$. Then for any integer $z$ relatively prime to $p$ we have

$$
K(q, m+n \phi(q)+1, z)=\left\{\begin{array}{c}
q^{n \phi(q) / 2} K\left(q, m+1, z\left(1-n p^{a-1}\right)\right) \\
\text { if } 2 \mid a \text { or if } p=2, a \geq 5, \text { and } 2 \nmid a, \\
q^{n \phi(q) / 2} \varepsilon_{p}^{n \phi(q)} K\left(q, m+1, z\left(1-n p^{a-1}\right)\right) \\
\text { if } p>2 \text { and } 2 \nmid a .
\end{array}\right.
$$

This Corollary simplifies hyper-Kloosterman sums of prime power moduli with larger $m, p \nmid m$, to hyper-Kloosterman sums with $m$ between 1 and $\phi(q)-1$. Consequently, the bounds for hyper-Kloosterman sums of prime power moduli proved by Dąbrowski and Fisher [1] (see (19) and (20) in Section 4) can be rewritten and improved for large $m$ when $p \nmid m$. These improved bounds may also be proved directly following their Theorem 1.8 and Example 1.17:

$$
\begin{aligned}
& |K(q, m+1, z)| \\
& \quad \leq \begin{cases}(r+1) q^{m / 2} & \text { if } p \nmid(r+1), \\
(r+1) p^{-h / 2} q^{m / 2} & \text { if } h \geq 1 \text { and } a \geq 3 h+2, \\
(r+1, p-1) p^{\min (h, a / 2-1)} q^{m / 2} & \text { if } h \geq 1 \text { and } 2 \mid a, \\
(r+1, p-1) p^{\min (h+1 / 2, a / 2-1)} q^{m / 2} & \text { if } h \geq 1 \text { and } 2 \nmid a,\end{cases}
\end{aligned}
$$

when $p>2$, and

$$
|K(q, m+1, z)| \leq \begin{cases}(r+1) p^{1-h / 2} q^{m / 2} & \text { if } h \geq 1 \text { and } a \geq 3 h+5, \\ p^{\min (h+1, a / 2-1)} q^{m / 2} & \text { if } h \geq 1 \text { and } 2 \mid a, \\ p^{\min (h+3 / 2, a / 2-1)} q^{m / 2} & \text { if } h \geq 1 \text { and } 2 \nmid a,\end{cases}
$$

when $p=2$, where $h$ is given by $p^{h} \|(r+1)$ and $m \equiv r(\bmod \phi(q))$ with $1 \leq r<\phi(q)$ and $p \nmid r$.

Using the Corollary and the identities in Theorem 3 backward, we can further deduce new bounds for hyper-Kloosterman sums from the bounds for the exponential sum $S_{k}(q, b, c)$. These new bounds are sharper than the improved bounds of Dąbrowski and Fisher in (1) and (2) when $m \equiv r$ $(\bmod \phi(q))$ with $r$ being less than and close to $\phi(q)-a$. Here in order to 
have

$$
\sum_{\substack{x \bmod q \\ p \mid x}} \mathrm{e}\left(\frac{b x+c x^{\phi(q)-r}}{q}\right)=0
$$

we need to assume that $\phi(q)-r \geq a$.

Theorem 4. Let $p$ be any prime. Assume that $a \geq 2$ when $p>2$ and $a \geq 4$ when $p=2$. Set $q=p^{a}$ and let $m$ be any positive integer with $p \nmid m$, $m \equiv r(\bmod \phi(q))$ and $1 \leq r \leq \phi(q)-a$. Define $h$ by $p^{h} \|(r+1)$. Then for any integer $z$ relatively prime to $p$ we have

$|K(q, m+1, z)|$

$$
\leq \begin{cases}(\phi(q)-r-1) q^{m / 2} & \text { if } p \nmid(r+1), \\ (\phi(q)-r-1) p^{-h / 2} q^{m / 2} & \text { if } h \geq 1 \text { and } a \geq 3 h+2, \\ (r+1, p-1) p^{\min (h, a / 2-1)} q^{m / 2} & \text { if } h \geq 1 \text { and } 2 \mid a, \\ (r+1, p-1) p^{\min (h+1 / 2, a / 2-1)} q^{m / 2} & \text { if } h \geq 1 \text { and } 2 \nmid a,\end{cases}
$$

when $p>2$, and

$$
|K(q, m+1, z)| \leq \begin{cases}(\phi(q)-r-1) p^{1-h / 2} q^{m / 2} & \text { if } h \geq 1 \text { and } a \geq 3 h+5, \\ p^{\min (h+1, a / 2-1)} q^{m / 2} & \text { if } h \geq 1 \text { and } 2 \mid a \\ p^{\min (h+3 / 2, a / 2-1)} q^{m / 2} & \text { if } h \geq 1 \text { and } 2 \nmid a,\end{cases}
$$

when $p=2$.

Estimation of hyper-Kloosterman sums for prime moduli was proved by Deligne [2] and Katz [3]. It is interesting to see whether bounds like those in Theorem 4 can be established for hyper-Kloosterman sums modulo $p$.

2. New Davenport-Hasse identities for Gauss sums. Let $p$ be a prime and $m>1$ an integer with $p \nmid m$. Let $\chi$ be any ramified multiplicative character on the $p$-adic field $\mathbb{Q}_{p}$ with conductor exponent $a(\chi)=a$. Here $\chi$ is ramified if it is non-trivial on $R_{p}^{\times}$, the group of invertible elements of the ring of integers $R_{p}$ in $\mathbb{Q}_{p}$; for a ramified multiplicative character $\chi$ its conductor exponent, denoted by $a(\chi)$, is the smallest positive integer $a$ such that $\chi$ is trivial on $1+p^{a} R_{p}$. Let $\psi$ be an additive character of $\mathbb{Q}_{p}$ whose order is zero. Here the order of an additive character $\psi$, denoted by $n(\psi)$, is the largest integer $n$ such that the character $\psi$ is trivial on $p^{-n} R_{p}$.

For any additive character $\phi$ we define the local $\varepsilon$-factor as

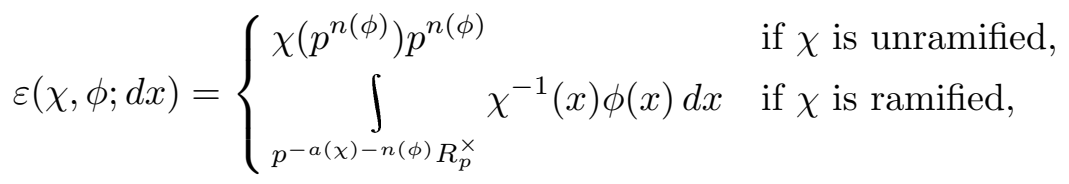

where $d x$ is a Haar measure on $\mathbb{Q}_{p}$ normalized by volume $\left(R_{p}\right)=1$. Then the new Davenport-Hasse identities for Gauss sums have the following form. 
TheOREM 5. Let $p$ be a prime and $m>1$ an integer with $p \nmid m$. Let $\psi$ be a non-trivial additive character of $\mathbb{Q}_{p}$ of order zero. Then for any ramified multiplicative character $\chi$ with conductor exponent $a(\chi)=a \geq 2$ we have

$$
(\varepsilon(\chi, \psi ; d x))^{m}= \begin{cases}q^{(m-1) / 2} \chi^{m}(m) \varepsilon\left(\chi^{m}, \psi ; d x\right) & \text { if } 2 \mid a, \\ q^{m-1-[m / p]} \chi^{m}(m) \varepsilon\left(\chi^{m}, \psi ; d x\right) & \\ \times \prod_{\substack{2 \leq j \leq m \\ p \nmid j(j-1)}} \int_{p^{(a-1) / 2} R_{p}} \chi\left(1+\frac{j-1}{2 j} y_{j}^{2}\right) d y_{j} & \text { if } 2 \nmid a,\end{cases}
$$

when $p>2$, and

$$
\begin{aligned}
& (\varepsilon(\chi, \psi ; d x))^{m} \\
& \quad= \begin{cases}q^{(m-1) / 2} \chi^{m}(m) \varepsilon\left(\chi^{m}, \psi ; d x\right) & \text { if } 2 \mid a, \\
q^{m-1-[(m-1) / 4]} \chi^{m}(m) \varepsilon\left(\chi^{m}, \psi ; d x\right) & \\
\times\left(\int_{u, v \in p^{(a-1) / 2} R_{p}} \chi\left(1+u^{2}+u v+v^{2}\right) d u d v\right)^{[(m+1) / 4]} & \text { if } 2 \nmid a,\end{cases}
\end{aligned}
$$

when $p=2$, where $q=p^{a}$.

Pr o of. Following the computation in Ye [9] and [10] we have

$$
(\varepsilon(\chi, \psi ; d x))^{m}=\int_{\left(q^{-1} R_{p}^{\times}\right)^{m}} \chi^{-1}\left(x_{1} \ldots x_{m}\right) \psi\left(x_{1}+\ldots+x_{m}\right) d x_{1} \ldots d x_{m} .
$$

Change variables from $x_{i}$ to $y_{i}=x_{i} / x_{1}$ for $i=2, \ldots, m$. Since $p \nmid m$, the conductor exponent of $\chi^{m}$ is still $a$. Consequently, the integral with respect to $x_{1}$ vanishes unless $1+y_{2}+\ldots+y_{m} \in R_{p}^{\times}$. Setting $z=x_{1}\left(1+y_{2}+\ldots+y_{m}\right)$ we get

$$
\begin{aligned}
(\varepsilon(\chi, \psi ; d x))^{m}= & q^{m-1} \varepsilon\left(\chi^{m}, \psi ; d x\right) \\
& \times \int_{\substack{y_{2}, \ldots, y_{m} \in R_{p}^{\times} \\
1+y_{2}+\ldots+y_{m} \in R_{p}^{\times}}} \chi\left(\frac{\left(1+y_{2}+\ldots+y_{m}\right)^{m}}{y_{2} \ldots y_{m}}\right) d y_{2} \ldots d y_{m} .
\end{aligned}
$$

Denote the integral by $I_{m}$. Since $a(\chi)=a \geq 2$, for $m \geq 3$ we set $y_{m}=y_{0}(1+u)$ where

$$
y_{0} \in\left(R_{p}^{\times}-\left(-\left(1+y_{2}+\ldots+y_{m-1}\right)+p R_{p}\right)\right) /\left(1+p^{[(a+1) / 2]} R_{p}\right)
$$

and $u \in p^{[(a+1) / 2]} R_{p}$. The integral with respect to $u$ vanishes unless $1+y_{2}+$ $\ldots+y_{m-1}-(m-1) y_{0} \in p^{[a / 2]} R_{p}$. Therefore the variables in $I_{m}$ satisfy

$$
1+y_{2}+\ldots+y_{m-1}-(m-1) y_{m} \in p^{[a / 2]} R_{p} \text {. }
$$


If $p \nmid(m-1)$, then we get the case discussed in [10]. Setting $y_{m}=\left(1+y_{2}+\right.$ $\left.\ldots+y_{m-1}\right) /(m-1)+y$ with $y \in p^{[a / 2]} R_{p}$ we get

$$
I_{m}=I_{m-1} \chi\left(\frac{m^{m}}{(m-1)^{m-1}}\right) \int_{p^{[a / 2]} R_{p}} \chi\left(1+\frac{(m-1) y^{2}}{2 m}\right) d y
$$

when $p>2, m \geq 3$, and $p \nmid m(m-1)$. When $a$ is even, we can further compute the integral in (4) to get

$$
I_{m}=q^{-1 / 2} \chi\left(\frac{m^{m}}{(m-1)^{m-1}}\right) I_{m-1}
$$

when $p>2, m \geq 3, p \nmid m(m-1)$, and $2 \mid a$.

Now we consider the case of $p \mid(m-1)$ and $m \geq 4$. Then from (3) we know that $1+y_{2}+\ldots+y_{m-1} \in p R_{p}$; hence $1+y_{2}+\ldots+y_{m-2} \in R_{p}^{\times}$ and $y_{m-1} \in-\left(1+y_{2}+\ldots+y_{m-2}\right)+(m-1) y_{m}+p^{[a / 2]} R_{p}$. Set $y_{m-1}=$ $-\left(1+y_{2}+\ldots+y_{m-2}\right)+(m-1) y_{m}+y$ with $y \in p^{[a / 2]} R_{p}$. Then

$$
\begin{gathered}
I_{m} \\
\int_{\substack{y \in p^{[a / 2]} R_{p} \\
y_{2}, \ldots, y_{m-2}, y_{m} \in R_{p}^{\times} \\
1+y_{2}+\ldots+y_{m-2} \in R_{p}^{\times}}} \chi\left(-\frac{\left(m y_{m}+y\right)^{m}}{y_{2} \ldots y_{m-2} y_{m}\left(1+y_{2}+\ldots+y_{m-2}-(m-1) y_{m}-y\right)}\right) \\
\quad \times d y d y_{2} \ldots d y_{m-2} d y_{m} .
\end{gathered}
$$

When $2 \mid a$, the integrand above equals

$$
\begin{aligned}
\chi\left(-\frac{m^{m} y_{m}^{m-1}}{y_{2} \ldots y_{m-2}\left(1+y_{2}+\ldots+y_{m-2}-(m-1) y_{m}\right)}\right) \\
\quad \times \chi\left(1+\left(\frac{1}{y_{m}}+\frac{1}{1+y_{2}+\ldots+y_{m-2}-(m-1) y_{m}}\right) y\right) .
\end{aligned}
$$

Consequently, in order to have a non-zero integral with respect to $y$ we must have

$$
\frac{1}{y_{m}}+\frac{1}{1+y_{2}+\ldots+y_{m-2}-(m-1) y_{m}} \in p^{a / 2} R_{p},
$$

which is equivalent to $1+y_{2}+\ldots+y_{m-2}-(m-2) y_{m} \in p^{a / 2} R_{p}$. Note that $p \nmid(m-2)$; hence we can set $y_{m}=\left(1+y_{2}+\ldots+y_{m-2}\right) /(m-2)+z$ with $z \in p^{a / 2} R_{p}$. Integrating with respect to $y$ and substituting the above expression of $y_{m}$ into

$$
\chi\left(-\frac{m^{m} y_{m}^{m-1}}{y_{2} \ldots y_{m-2}\left(1+y_{2}+\ldots+y_{m-2}-(m-1) y_{m}\right)}\right)
$$


we can see that the resulting expression is independent of $z$ :

$$
\chi\left(\frac{m^{m}}{(m-2)^{m-2}}\right) \chi\left(\frac{\left(1+y_{2}+\ldots+y_{m-2}\right)^{m-2}}{y_{2} \ldots y_{m-2}}\right) .
$$

Integrating with respect to $z$ we get

$$
I_{m}=q^{-1} \chi\left(\frac{m^{m}}{(m-2)^{m-2}}\right) I_{m-2}
$$

when $p>2, m \geq 4, p \nmid m, p \mid(m-1), a \geq 2$, and $2 \mid a$.

Now let us turn to the case of $2 \nmid a$. Then the integral in (6) becomes

$$
\begin{aligned}
&= \int_{\substack{y \in p^{(a-1) / 2} R_{p} \\
y_{2}, \ldots, y_{m-2}, y_{m} \in R_{p}^{\times} \\
1+y_{2}+\ldots+y_{m-2} \in R_{p}^{\times}}} \chi\left(-\frac{m^{m} y_{m}^{m-1}}{y_{2} \ldots y_{m-2}\left(1+y_{2}+\ldots+y_{m-2}-(m-1) y_{m}\right)}\right) \\
& \times \chi\left(1+y\left(\frac{1}{y_{m}}+\frac{1}{1+y_{2}+\ldots+y_{m-2}-(m-1) y_{m}}\right)\right. \\
&+\left.y^{2}\left(\frac{m-1}{2 m y_{m}^{2}}+\frac{\frac{1}{y_{m}}+\frac{1}{1+y_{2}+\ldots+y_{m-2}-(m-1) y_{m}}}{1+y_{2}+\ldots+y_{m-2}-(m-1) y_{m}}\right)\right) \\
& \times d y d y_{2} \ldots d y_{m-2} d y_{m} .
\end{aligned}
$$

Since we assume in this case that $p>2$, the term $(m-1) /\left(2 m y_{m}^{2}\right) \in p R_{p}$ and hence can be taken out of the above integrand. Setting $y=z+u$ with $z \in p^{(a-1) / 2} R_{p} / p^{(a+1) / 2} R_{p}$ and $u \in p^{(a+1) / 2} R_{p}$, we have $y^{2} \in z^{2}+q R_{p}$. Integrating with respect to $u$ we get a non-zero result only if

$$
\frac{1}{y_{m}}+\frac{1}{1+y_{2}+\ldots+y_{m-2}-(m-1) y_{m}} \in p^{(a-1) / 2} R_{p}
$$

Because of this condition, the integrand in (8) can be simplified to

$$
\begin{aligned}
\chi\left(-\frac{m^{m} y_{m}^{m-1}}{y_{2} \ldots y_{m-2}\left(1+y_{2}+\ldots+y_{m-2}-(m-1) y_{m}\right)}\right) \\
\quad \times \chi\left(1+y\left(\frac{1}{y_{m}}+\frac{1}{1+y_{2}+\ldots+y_{m-2}-(m-1) y_{m}}\right)\right) .
\end{aligned}
$$

Then the integral with respect to $y$ is non-zero only when

$$
\frac{1}{y_{m}}+\frac{1}{1+y_{2}+\ldots+y_{m-2}-(m-1) y_{m}} \in p^{(a+1) / 2} R_{p}
$$

i.e., only when $1+y_{2}+\ldots+y_{m-2}-(m-2) y_{m} \in p^{(a+1) / 2} R_{p}$. Integrate with respect to $y$ and set $y_{m}=\left(1+y_{2}+\ldots+y_{m-2}\right) /(m-2)+z$ with $z \in p^{(a+1) / 2} R_{p}$. If we substitute this expression for $y_{m}$, we can see the 
integrand is indeed independent of $z$. Integrating with respect to $z$ as before we conclude that

$$
I_{m}=q^{-1} \chi\left(\frac{m^{m}}{(m-2)^{m-2}}\right) I_{m-2}
$$

when $p>2, m \geq 4, p \nmid m, p \mid(m-1), a \geq 2$, and $2 \nmid a$.

Using the same approach as above we can also get

$$
I_{2}=q^{-1 / 2} \chi\left(2^{2}\right)
$$

when $p>2, a \geq 2$, and $2 \mid a$, and

$$
I_{2}=\chi\left(2^{2}\right) \int_{p^{(a-1) / 2} R_{p}} \chi\left(1+\frac{y^{2}}{4}\right) d y
$$

when $p>2, a \geq 2$, and $2 \nmid a$. Putting all these results from (4), (5), (7), (9), (10), and (11) together we get the following expressions for $I_{m}$ :

$$
I_{m}=q^{(1-m) / 2} \chi\left(m^{m}\right)
$$

when $p>2, m \geq 2, p \nmid m, a \geq 2$, and $2 \mid a$, and

$$
I_{m}=q^{-[m / p]} \chi\left(m^{m}\right) \prod_{\substack{2 \leq j \leq m \\ p \nmid j(j-1)}} \int_{p^{(a-1) / 2} R_{p}} \chi\left(1+\frac{(j-1) y_{j}^{2}}{2 j}\right) d y_{j}
$$

when $p>2, m \geq 2, p \nmid m, a \geq 2$, and $2 \nmid a$. Theorem 5 in the case of $p>2$ then follows.

We now consider the case of $p=2$. Following the same approach as above we set

$$
I_{m}=\int_{\substack{y_{2}, \ldots, y_{m} \in R_{p}^{\times} \\ 1+y_{2}+\ldots+y_{m} \in R_{p}^{\times}}} \chi\left(\frac{\left(1+y_{2}+\ldots+y_{m}\right)^{m}}{y_{2} \ldots y_{m}}\right) d y_{2} \ldots d y_{m}
$$

so that

$$
(\varepsilon(\chi, \psi ; d x))^{m}=q^{m-1} \varepsilon\left(\chi^{m}, \psi ; d x\right) I_{m} .
$$

Since $p=2$ and $2 \nmid m$, we always have $p \mid(m-1)$. For $m \geq 5$ we get the same expression of $I_{m}$ as in (6) which implies (7) when $a$ is even. When $a$ is odd, we get (8) again. If $4 \mid(m-1)$, then we still have $(m-1) /\left(2 m y_{m}^{2}\right) \in p R_{p}$ and hence this term can be taken out of the integrand in (8). By the same computation, we get (9). Therefore

$$
I_{m}=q^{-1} \chi\left(\frac{m^{m}}{(m-2)^{m-2}}\right) I_{m-2}
$$

when (i) $p=2, m \geq 5,2 \nmid m, a \geq 2$, and $2 \mid a$, or (ii) $p=2, m \geq 5,2 \nmid m$, $4 \mid(m-1), a \geq 3$, and $2 \nmid a$. 
Now we consider the case of $p=2,2 \nmid m, 4 \nmid(m-1), a \geq 3$, and $2 \nmid a$. Then $(m-1) /\left(2 m y_{m}^{2}\right) \in R_{p}^{\times}$. Consequently, by setting $y=z+u$ with $z \in p^{(a-1) / 2} R_{p} / p^{(a+1) / 2} R_{p}$ and $u \in p^{(a+1) / 2} R_{p}$, we can only get

$$
\frac{1}{y_{m}}+\frac{1}{1+y_{2}+\ldots+y_{m-2}-(m-1) y_{m}} \in p^{(a-1) / 2} R_{p} .
$$

Set $y_{m}=\left(1+y_{2}+\ldots+y_{m-2}\right) /(m-2)+z$ with $z \in p^{(a-1) / 2} R_{p}$. Then (8) can be simplified to

$$
\begin{aligned}
I_{m}= & \int_{\substack{y, z \in p^{(a-1) / 2} R_{p} \\
y_{2}, \ldots, y_{m-2} \in R_{p}^{\times} \\
1+y_{2}+\ldots+y_{m-2} \in R_{p}^{\times}}} \chi\left(\frac{m^{m}}{(m-2)^{m-2}}\right) \chi\left(\frac{\left(1+y_{2}+\ldots+y_{m-2}\right)^{m-2}}{y_{2} \ldots y_{m-2}}\right) \\
& \times \chi\left(1+\frac{(m-1) z^{2}}{2(m-2)}\right) \chi\left(1-y z+\frac{(m-1) y^{2}}{2 m}\right) d y d z d y_{2} \ldots d y_{m-2} .
\end{aligned}
$$

Since $(m-1) / 2$ is an odd integer we can further simplify the integrals with respect to $y$ and $z$ to get

$$
I_{m}=\chi\left(\frac{m^{m}}{(m-2)^{m-2}}\right) I_{m-2} \int_{y, z \in p^{(a-1) / 2} R_{p}} \chi\left(1+y^{2}+y z+z^{2}\right) d y d z
$$

when $p=2, m \geq 5,2 \nmid m, 4 \nmid(m-1), a \geq 3$, and $2 \nmid a$.

We can also compute $I_{3}$ :

$$
I_{3}=q^{-1} \chi\left(3^{3}\right)
$$

if $p=2, a \geq 2$, and $2 \mid a$, and

$$
I_{3}=\chi\left(3^{3}\right) \int_{u, v \in p^{(a-1) / 2} R_{p}} \chi\left(1+u^{2}+u v+v^{2}\right) d u d v
$$

if $p=2, a \geq 2$, and $2 \nmid a$. Putting the results in (12)-(15) together we prove Theorem 5 for $p=2$.

3. Identities for hyper-Kloosterman sums. In this section we will prove Theorem 3 when $a \geq 3$ is odd. Denote the hyper-Kloosterman sum over $p$-adic field by

$$
K_{p}(q, m+1, z)=\sum_{x_{1}, \ldots, x_{m} \in R_{p}^{\times} /\left(1+q R_{p}\right)} \psi\left(\frac{1}{q}\left(x_{1}+\ldots+x_{m}+\frac{z}{x_{1} \ldots x_{m}}\right)\right) .
$$

Applying the Mellin transform to the $p$-adic hyper-Kloosterman sum as in [10], we get

$$
\int_{R_{p}^{\times}} \chi^{-1}(z) K_{p}(q, m+1, z) d z=q^{-1} \chi^{-(m+1)}(q)(\varepsilon(\chi, \psi ; d x))^{m+1}
$$


when $a(\chi)=a \geq 2$ and $q=p^{a}$. By Theorem 5 when $p$ is odd and $p \nmid m$ the above becomes

$$
\begin{aligned}
q^{m-2-[m / p]} \chi^{-(m+1)}(q) \chi^{m}(m) \varepsilon(\chi, \psi ; d x) \varepsilon\left(\chi^{m}, \psi ; d x\right) & \\
\times & \prod_{\substack{2 \leq j \leq m \\
p \nmid j(j-1)}} \int_{p^{(a-1) / 2} R_{p}} \chi\left(1+\frac{j-1}{2 j} y_{j}^{2}\right) d y_{j}
\end{aligned}
$$

if $a$ is odd. By the same computation as in [10] we can prove that

$$
\begin{aligned}
\int_{R_{p}^{\times}} & \chi^{-1}(z) K_{p}(q, m+1, z) d z \\
= & q^{(m-1) / 2} \varepsilon_{p}^{m-1-2[m / p]} \int_{R_{p}^{\times}} \chi^{-1}(z) d z \\
& \times \sum_{x \in R_{p}^{\times} /\left(1+q R_{p}\right)} \psi\left(\frac{1}{q}\left(m x+\frac{z}{x^{m}}\right)\right) \prod_{\substack{2 \leq j \leq m \\
p \nmid j(j-1)}}\left(\frac{2 j(j-1) x^{m} z}{p}\right)
\end{aligned}
$$

when $p>2, p \nmid m, 2 \nmid a$ for any multiplicative character $\chi$. Since the number of factors in the product in (16) is $m-1-2[m / p]$, the product equals

$$
\begin{gathered}
\left(\frac{x^{m}}{p}\right)^{m-1-2[m / p]}\left(\frac{z}{p}\right)^{m-1-2[m / p]} \prod_{\substack{2 \leq j \leq m \\
p \nmid j(j-1)}}\left(\frac{j(j-1)}{p}\right) \\
=\left(\frac{x}{p}\right)^{m(m-1)}\left(\frac{2 z}{p}\right)^{m-1}\left(\frac{m}{p}\right) \prod_{1 \leq k<m / p}\left(\frac{(k p+1)(k p-1)}{p}\right) \\
=\left(\frac{2 z}{p}\right)^{m-1}\left(\frac{m}{p}\right)\left(\frac{-1}{p}\right)^{[m / p]} .
\end{gathered}
$$

Consequently, we proved the following identity over the $p$-adic field:

$$
\begin{aligned}
K_{p}(q, m+1, z)= & q^{(m-1) / 2} \varepsilon_{p}^{m-1}\left(\frac{2^{m-1} z^{m-1} m}{p}\right) \\
& \times \sum_{x \in R_{p}^{\times} /\left(1+q R_{p}\right)} \psi\left(\frac{1}{q}\left(m x+\frac{z}{x^{m}}\right)\right)
\end{aligned}
$$

when $p>2, p \nmid m, 2 \nmid a$, and $p \nmid z$. This identity is equivalent to Theorem 3 in the case of odd $p$ which is a generalization of a result proved in [10].

Now we turn to the case of $p=2$ with $2 \nmid m$. When $a \geq 3$ is odd, we deduce from Theorem 5 that 


$$
\begin{aligned}
\int_{R_{p}^{\times}} \chi^{-1}(z) & K_{p}(q, m+1, z) d z \\
= & q^{m-2-[(m-1) / 4]} \chi^{-(m+1)}(q) \chi^{m}(m) \varepsilon(\chi, \psi ; d x) \varepsilon\left(\chi^{m}, \psi ; d x\right) \\
& \times\left(\int_{u, v \in p^{(a-1) / 2} R_{p}} \chi\left(1+u^{2}+u v+v^{2}\right) d u d v\right)^{[(m+1) / 4]} .
\end{aligned}
$$

We have

$$
\begin{aligned}
q^{-2} \chi^{-(m+1)}(q) & \varepsilon(\chi, \psi ; d x) \varepsilon\left(\chi^{m}, \psi ; d x\right) \\
& =q^{-2} \chi^{-(m+1)}(q) \int_{\left(q^{-1} R_{p}^{\times}\right)^{2}} \chi^{-1}\left(x_{1} x_{2}^{m}\right) \psi\left(x_{1}+x_{2}\right) d x_{1} d x_{2} \\
& =\int_{\left(R_{p}^{\times}\right)^{2}} \chi^{-1}(x) \psi\left(\frac{1}{q}\left(y+\frac{x}{y^{m}}\right)\right) d x d y .
\end{aligned}
$$

Rewriting the power in (17) as

$$
\prod_{1 \leq j \leq[(m+1) / 4]} \int_{u_{j}, v_{j} \in p^{(a-1) / 2} R_{p}} \chi\left(1+u_{j}^{2}+u_{j} v_{j}+v_{j}^{2}\right) d u_{j} d v_{j}
$$

we then change variables from $x$ to $z$ via

$$
x=z m^{m} \prod_{1 \leq j \leq[(m+1) / 4]}\left(1+u_{j}^{2}+u_{j} v_{j}+v_{j}^{2}\right) .
$$

Then the expression on the right side of (17) becomes

$$
\begin{aligned}
& q^{m-[(m-1) / 4]} \int_{\left(R_{p}^{\times}\right)^{2}} \chi^{-1}(z) d z d y \\
& \times \int_{\substack{u_{j}, v_{j} \in p^{(a-1) / 2} R_{p} \\
1 \leq j \leq[(m+1) / 4]}} \psi\left(\frac{1}{q}\left(y+\frac{z m^{m}}{y^{m}} \prod_{\substack{1 \leq j \leq[(m+1) / 4]\\
}}\left(1+u_{j}^{2}+u_{j} v_{j}+v_{j}^{2}\right)\right)\right) \\
& \times d z d y d u_{1} d v_{1} \ldots d u_{[(m+1) / 4]} d v_{[(m+1) / 4]} .
\end{aligned}
$$

Changing variables again and multiplying out the product we get

$$
\begin{aligned}
q^{m-1-[(m-1) / 4]} \int_{R_{p}^{\times}} \chi^{-1}(z) d z \sum_{y \in R_{p}^{\times} /\left(1+q R_{p}\right)} \psi\left(\frac{1}{q}\left(m y+\frac{z}{y^{m}}\right)\right) \\
\times\left(\int_{u, v \in p^{(a-1) / 2} R_{p}} \psi\left(\frac{z}{q y^{m}}\left(u^{2}+u v+v^{2}\right)\right) d u d v\right)^{[(m+1) / 4]} .
\end{aligned}
$$

In order to compute the integral with respect to $u$ and $v$ we write it as a 
finite sum

$$
\begin{aligned}
\int_{u, v \in p^{(a-1) / 2} R_{p}} & \psi\left(\frac{z}{q y^{m}}\left(u^{2}+u v+v^{2}\right)\right) d u d v \\
= & p^{-a-1} \sum_{u, v \in p^{(a-1) / 2} R_{p} / p^{(a+1) / 2} R_{p}} \psi\left(\frac{z}{q y^{m}}\left(u^{2}+u v+v^{2}\right)\right) \\
= & p^{-a-1} \sum_{u, v \in R_{p} / p R_{p}} \psi\left(\frac{z}{p y^{m}}\left(u^{2}+u v+v^{2}\right)\right) .
\end{aligned}
$$

Since $p=2$, we can take $u, v=0,1$ and get

$$
p^{-a-1}\left(1+2 \psi\left(\frac{z}{p y^{m}}\right)+\psi\left(\frac{3 z}{p y^{m}}\right)\right) .
$$

Since the order of $\psi$ is zero and $p^{-1} R_{p}^{\times} / R_{p}$ has only one element, we have

$$
\psi\left(\frac{z}{p y^{m}}\right)=\psi\left(\frac{3 z}{p y^{m}}\right)=-1
$$

and hence

$$
\int_{u, v \in p^{(a-1) / 2} R_{p}} \psi\left(\frac{z}{q y^{m}}\left(u^{2}+u v+v^{2}\right)\right) d u d v=-q^{-1} .
$$

Consequently,

$$
\begin{aligned}
& \int_{R_{p}^{\times}} \chi^{-1}(z) K_{p}(q, m+1, z) d z \\
& \quad=q^{(m-1) / 2}\left(\frac{2}{m}\right) \int_{R_{p}^{\times}} \chi^{-1}(z) d z \sum_{y \in R_{p}^{\times} /\left(1+q R_{p}\right)} \psi\left(\frac{1}{q}\left(m y+\frac{z}{y^{m}}\right)\right)
\end{aligned}
$$

for any ramified character $\chi$ with conductor exponent $a(\chi)=a$, where we used the facts that

$$
[(m-1) / 4]+[(m+1) / 4]=(m-1) / 2 \quad \text { and } \quad(-1)^{[(m+1) / 4]}=\left(\frac{2}{m}\right) .
$$

Since $a>1$ this identity also holds for other multiplicative character $\chi$. Therefore we proved the following identity which is equivalent to Theorem 3 in the case of $p=2, p \nmid m, a \geq 3$, and $2 \nmid a$ :

$$
K_{p}(q, m+1, z)=q^{(m-1) / 2}\left(\frac{2}{m}\right) \sum_{y \in R_{p}^{\times} /\left(1+q R_{p}\right)} \psi\left(\frac{1}{q}\left(m y+\frac{z}{y^{m}}\right)\right)
$$

for any $z \in R_{p}^{\times}$. 
4. Estimation of exponential sums. We first prove Theorem 2 using Theorem 1.8 of Dąbrowski and Fisher [1]. Let $f(x)=b x+c x^{k}$ be a polynomial with $b, c$, and $k$ relatively prime to $p$. For $a \geq 2$ we set $q=p^{a}$ and $j=[a / 2]$. Define the scheme $D$ of critical points of $f$ as zeros of $f^{\prime}(x)=b+c k x^{k-1}$. Then a point $x$ in $D$ is étale if $p \nmid(k-1), x$ is h-étale if $p^{h} \|(k-1)$, and $x$ is strictly $h$-étale if $p^{h+1} \|(k-1)$. Theorem 1.8(a) of Dąbrowski and Fisher [1] says that

$$
\left|S_{k}(q, b, c)\right| \leq \begin{cases}\left|D\left(\mathbb{Z} / p^{j} \mathbb{Z}\right)\right| q^{1 / 2} & \text { if } 2 \mid a \text { or if } 2 \nmid a \text { and } p \nmid(k-1), \\ \left|D\left(\mathbb{Z} / p^{j} \mathbb{Z}\right)\right| p^{1 / 2} q^{1 / 2} & \text { if } 2 \nmid a \text { and } p \mid(k-1) .\end{cases}
$$

Theorem 1.8(b) on the other hand implies that

$$
\left|S_{k}(q, b, c)\right| \leq\left|D\left(\mathbb{Z}_{p}\right)\right| p^{h / 2} q^{1 / 2}
$$

if $a \geq 3 h+2$ when $p>2$ or $a \geq 3 h+5$ when $p=2$, where $h \geq 1$ is given by $p^{h} \|(k-1)$. Following Example 1.17 of [1] we have

$$
\left|D\left(\mathbb{Z} / p^{j} \mathbb{Z}\right)\right| \leq \begin{cases}k-1 & \text { if } p \nmid(k-1), \\ p^{\min (h+1, j-1)} & \text { if } p=2 \text { and } h \geq 1, \\ (k-1, p-1) p^{\min (h, j-1)} & \text { if } p>2 \text { and } h \geq 1,\end{cases}
$$

and

$$
\left|D\left(\mathbb{Z}_{p}\right)\right| \leq \begin{cases}(k-1) p^{-h} & \text { if } p>2 \\ (k-1) p^{1-h} & \text { if } p=2 .\end{cases}
$$

Substituting these results into the above inequalities for the exponential sum, we get the estimates in Theorem 2 .

By similar computation the bounds for the hyper-Kloosterman sum $K(q, m+1, z)$ considered in Example 1.17 of Dąbrowski and Fisher [1] can be written in the following way. Here $h$ is given by $p^{h} \|(m+1)$.

$$
\begin{aligned}
& |K(q, m+1, z)| \\
& \leq \begin{cases}(m+1) q^{m / 2} & \text { if } p \nmid(m+1), \\
(m+1) p^{-h / 2} q^{m / 2} & \text { if } h \geq 1 \text { and } a \geq 3 h+2, \\
(m+1, p-1) p^{\min (h, a / 2-1)} q^{m / 2} & \text { if } h \geq 1 \text { and } 2 \mid a, \\
(m+1, p-1) p^{\min (h+1 / 2, a / 2-1)} q^{m / 2} & \text { if } h \geq 1 \text { and } 2 \nmid a,\end{cases}
\end{aligned}
$$

when $p>2$, and

$$
|K(q, m+1, z)| \leq \begin{cases}(m+1) p^{1-h / 2} q^{m / 2} & \text { if } h \geq 1 \text { and } a \geq 3 h+5, \\ p^{\min (h+1, a / 2-1)} q^{m / 2} & \text { if } h \geq 1 \text { and } 2 \mid a \\ p^{\min (h+3 / 2, a / 2-1)} q^{m / 2} & \text { if } h \geq 1 \text { and } 2 \nmid a,\end{cases}
$$

when $p=2$. As before here we assume that $p \nmid m$. By the identities of hyperKloosterman sums in Theorem 3, we get the same bounds as in Theorem 1 
but for the exponential sum

$$
\sum_{\substack{x \bmod q \\(x, p)=1}} \mathrm{e}\left(\frac{m x+z \bar{x}^{m}}{q}\right)
$$

if we set $k=\phi(q)-m$. For $m$ in the range of $1 \leq m \leq \phi(q)-a$, however

$$
\sum_{\substack{x \bmod q \\ p \mid x}} \mathrm{e}\left(\frac{m x+z x^{\phi(q)-m}}{q}\right)=0 .
$$

This completes the proof of Theorem 1.

Acknowledgements. The author would like to thank the referee for helpful suggestions.

\section{References}

[1] R. Dąbrowski and B. Fisher, A stationary phase formula for exponential sums over $\mathbb{Z} / p^{m} \mathbb{Z}$ and applications to GL(3)-Kloosterman sums, Acta Arith. 80 (1997), $1-48$.

[2] P. Deligne, Applications de la formule des traces aux sommes trigonométriques, in: Cohomologie Etale (SGA 4 1/2), Lecture Notes in Math. 569, Springer, Berlin, 1977, 168-232.

[3] N. M. Katz, Gauss Sums, Kloosterman Sums, and Monodromy Groups, Ann. of Math. Stud. 116, Princeton Univ. Press, Princeton, 1988.

[4] -, Exponential Sums and Differential Equations, Ann. of Math. Stud. 124, Princeton Univ. Press, Princeton, 1990.

[5] J. H. Loxton and R. A. Smith, On Hua's estimate for exponential sums, J. London Math. Soc. 26 (1982), 15-20.

[6] J. H. Loxton and R. C. Vaughan, The estimation of complete exponential sums, Canad. Math. Bull. 28 (1985), 440-454.

[7] R. A. Smith, On n-dimensional Kloosterman sums, J. Number Theory 11 (1979), 324-343.

[8] R. C. Vaughan, The Hardy-Littlewood Method, 2nd ed., Cambridge Tracts in Math. 125, Cambridge Univ. Press, Cambridge, 1997.

[9] Y. Ye, The lifting of an exponential sum to a cyclic algebraic number field of a prime degree, Trans. Amer. Math. Soc. 350 (1998), 5003-5015.

[10] - Hyper-Kloosterman sums and estimation of exponential sums of polynomials of higher degrees, Acta Arith. 86 (1998), 255-267.

Department of Mathematics

The University of Iowa

Iowa City, IA 52242-1419

U.S.A.

E-mail: yey@math.uiowa.edu 